Number of pages: 26

Number of tables: 2

Number of figures: 9

Number of references: 15

\title{
The vibration discomfort of standing people:
}

\section{evaluation of multi-axis vibration}

\author{
Olivier Thuong and Michael J Griffin \\ Human Factors Research Unit \\ Institute of Sound and Vibration Research \\ University of Southampton, SO17 1BJ \\ United Kingdom
}

Correspondence address:

Professor Michael J Griffin

Human Factors Research Unit

Institute of Sound and Vibration Research

University of Southampton

Southampton SO17 1BJ

England

Telephone: (+44) 02380592277

Facsimile: (+44) 02380592927

E-mail: M.J.Griffin@soton.ac.uk 


\begin{abstract}
Few studies have investigated discomfort caused by multi-axis vibration and none has explored methods of predicting the discomfort of standing people from simultaneous fore-and-aft, lateral, and vertical vibration of a floor. Using the method of magnitude estimation, 16 subjects estimated their discomfort caused by dual-axis and tri-axial motions (octave-bands centred on either $1 \mathrm{~Hz}$ or $4 \mathrm{~Hz}$ with various magnitudes in the fore-and-aft, lateral, and vertical directions) and the discomfort caused by single-axis motions. The method of predicting discomfort assumed in current standards (square-root of the sums of squares of the three components weighted according to their individual contributions to discomfort) provided reasonable predictions of the discomfort caused by multi-axis vibration. Improved predictions can be obtained for specific stimuli, but no single simple method will provide accurate predictions for all stimuli because the rate of growth of discomfort with increasing magnitude of vibration depends on the frequency and direction of vibration.
\end{abstract}

\title{
Practitioner summary ( $<50$ words)
}

Useful estimates of the vibration discomfort of standing people can be obtained from the root-sums-of squares of the floor acceleration in each of the three directions (fore-andaft, lateral, and vertical) if the three components are frequency-weighted according to the dependence of discomfort on the frequency of vibration in each axis.

\section{Keywords:}

Whole-body vibration; standing; multi-axis vibration; discomfort. 


\section{Introduction}

People are exposed to vibration in many forms of transport. While it is often possible to sit, there are many situations where it is necessary to stand during all, or part, of a journey. The discomfort caused by vibration has been studied for seated people and standards have been evolved. There has been less investigation of the discomfort caused by the vibration of standing people.

Studies with seated people have mostly investigated how vibration discomfort depends on the magnitude, frequency, waveform, and duration of vertical vibration (e.g., Donati et al. 1983; Parsons and Griffin, 1988; Ruffell and Griffin, 1995; Morioka and Griffin, 2006). Some studies have explored the discomfort of seated people exposed to non-vertical vibration (e.g., Griffin et al., 1982; Morioka and Griffin, 2006), and some have investigated methods of predicting the vibration discomfort of standing people (Thuong and Griffin, 2011a,b,c, 2012). A few studies have investigated the discomfort caused by dual-axis vibration (e.g., Griffin and Whitham, 1977; Fairley and Griffin, 1988; Mistrot et al., 1990; Griefahn and Brode, 1999) but there are no known systematic investigations of methods of predicting the discomfort caused by the tri-axial vibration of either seated or standing people.

Experimental studies have been conducted with seated people exposed to dual-axis sinusoidal vibration. The findings suggest that the discomfort caused by multi-axis vibration can be predicted from the square-root of the sums of the squares of the components in each axis, weighted according to their individual contributions to discomfort (e.g., Griffin and Whitham, 1977; Fairley and Griffin, 1988; Mistrot et al., 1990; Griefahn and Brode, 1999). This is the basis for British Standard 6841 (1987) and International Standard 2631-1 (1997) recommending the calculation of the root-sum-ofsquares when evaluating the severity of multi-axis vibration. An alternative method for 
predicting the discomfort caused by multi-axis vibration is based on a 'masking' model. It is assumed that the most uncomfortable vibration components reduce the effect of secondary components, so the total discomfort is the sum of the discomfort caused by the main component and a fraction of the discomfort caused by secondary components (e.g., Griffin and Whitham, 1977).

With standing people exposed to multi-axis vibration, this experiment was designed to determine a method of predicting their vibration discomfort (i.e., $\Psi_{\text {total }}$ ) from the discomfort (i.e., $\Psi_{x}, \psi_{y}$ and $\Psi_{z}$ ) caused by each of the single-axis components of the multi-axis motion.

\section{Method}

\subsection{Equipment}

Motions were produced by a simulator designed to expose people to fore-and-aft, lateral, vertical, roll, pitch, and yaw vibration with a maximum displacement of $\pm 250 \mathrm{~mm}$ in the fore-and-aft and lateral directions and $\pm 500 \mathrm{~mm}$ in the vertical direction (Figure 1). Although the simulator was capable of roll, pitch and yaw oscillation, only translational vibration was used in this study. The simulator was controlled by a Pulsar Digital Controller (Servotest Systems, Egham, UK). Motion stimuli were generated in Matlab (version 14009a) using the Matlab Toolbox HVLAB HRV (version 1.1) developed in the Human Factors Research Unit (University of Southampton).

\section{FIGURE 1 AROUND HERE}

The vibration was monitored using Setra $141 \mathrm{~A}$ capacitive accelerometers secured to the simulator table. The signals from the transducers were sampled by a Pulsar Digital Controller software at 256 samples per second after low pass filtering at $64 \mathrm{~Hz}$. 


\subsection{Motions}

Subjects were exposed to vibration stimuli with components in one, two, or three of the translational directions: fore-and-aft, lateral, and vertical. The vibration stimuli were 6-s periods of octave-band random vibration centred on either $1 \mathrm{~Hz}$ (i.e., in the range 0.7 to 1.4 Hz) or $4 \mathrm{~Hz}$ (i.e., in the range 2.8 to $5.6 \mathrm{~Hz}$ ). These waveforms were chosen because they have well-defined frequency content and are similar to the transient motions present in some transport environments.

Motion stimuli were presented in pairs: the first motion was called the 'reference motion' and the second was called the 'test motion'. Within a pair of stimuli, the reference motion and the test motion had the same frequency.

The reference motion was tri-axial vibration, composed of fore-and-aft, lateral, and vertical vibration with magnitudes as indicated in the first row of Table 1. For both frequencies, the reference motion had the same waveform throughout the experiment. The test stimuli were constructed with fore-and-aft, lateral, and vertical vibration having all possible combinations of the five magnitudes indicated in Table 1 . When the magnitude in one or two directions was zero, the test motion was, respectively, dual-axis or single-axis. Since the magnitude in each of the three axes was one of five preselected magnitudes (including 0 , being a possible magnitude), there were $5 \times 5 \times 5=125$ different stimuli. A stimuli with a magnitude of 0 in all directions is not of interest and was removed, therefore a total of 124 stimuli were presented with both frequencies. A different waveform was used for each magnitude and each direction of vibration. For a given magnitude and direction, the waveform was the same for all subjects.

TABLE 1 AROUND HERE 
The vibration magnitudes in each direction were chosen, based on previous results (Thuong and Griffin, 2012), so that at both frequencies they would cause approximately equivalent discomfort.

\subsection{Procedure}

Subjects attended two sessions, one for each frequency of vibration (i.e., 1 or $4 \mathrm{~Hz}$ ), during which they judged 124 test motions relative to either a $1-\mathrm{Hz}$ tri-axial reference motion or a $4-\mathrm{Hz}$ tri-axial reference motion. The stimuli were presented in a different randomized order to each subject.

After each pair of stimuli, subjects were asked to provide a number reflecting the discomfort caused by the test stimulus assuming the discomfort caused by the reference was 100. The vibration pair could be repeated if the subjects were unsure of their answer. No particular instruction was given to the subjects as to how to rate discomfort, or in which part of the body discomfort should be felt.

\subsection{Subjects}

Sixteen healthy male university students and staff with median age 25 years (range 19 to $30 \mathrm{y}$ ), stature $178 \mathrm{~cm}$ (165 to $198 \mathrm{~cm}$ ), weight $76 \mathrm{~kg}$ (50 to $104 \mathrm{~kg}$ ) participated in the study. They attended two sessions with each session lasting approximately 80 minutes.

The experiment was approved by the Human Experimentation Safety and Ethics Committee of the Institute of Sound and Vibration Research at the University of Southampton.

\subsection{Conditions and posture}

The subjects wore socks but no shoes and wore a loose harness in case they should fall

(Figure 1). The harness did not provide support or restrict movement when subjects 
stood as instructed. It was attached to an extruded aluminium frame secured to the simulator table.

Subjects maintained an upright posture, with their knees locked, and kept their eyes closed. Their feet were parallel and separated so that their lateral 'base of support' (distance between the outer edges of their feet) was $350 \mathrm{~mm}$ (i.e., the median shoulder width for adult males (Pheasant, 1988).

Subjects wore headphones delivering broadband noise at $65 \mathrm{~dB}(A)$ and providing some acoustic isolation from noises produced by the simulator, which were always less than $51 \mathrm{~dB}(\mathrm{~A})$ at the location of the subjects.

\subsection{Analysis}

Some of the motion stimuli had a magnitude of zero in two of the directions, so they were single-axis stimuli. In each of the three axes, the magnitude could take one of four non-zero values, so there were 12 single-axis motion stimuli. With both frequencies, subject judgments indicated the discomfort caused by these 12 single-axis motions. This provided the discomfort of each component in the 112 dual-axis or tri-axial motions at each frequency.

\subsubsection{Power summation methods}

Subject judgements of the discomfort caused by all multi-axis motions were compared with the discomfort predicted from the discomfort caused by the single-axis motions using a 'power summation' method:

$$
\psi_{\text {total }}=\left(\psi_{x}^{\alpha}+\psi_{y}^{\alpha}+\psi_{z}^{\alpha}\right)^{1 / \alpha}
$$

where:

$\Psi_{\text {total }}$ is the discomfort of the multi-axis motion 
$\Psi_{x}, \Psi_{y}$, and $\Psi_{z}$ are the discomfort caused by the fore-and-aft, lateral, and vertical components, respectively, when presented alone.

When $\alpha=1$ the discomfort of the multi-axis motion is predicted by the linear sum of the discomfort in each direction. When $\alpha=\infty$ the discomfort of the multi-axis motion is predicted by the discomfort of the most uncomfortable component

Assuming the predicted discomfort is greater than the measured discomfort when $\alpha=1$ and less than the measured discomfort when $\alpha=\infty$, there must be an intermediate value of $\alpha$ for which the discomfort predicted by Equation (1) does not differ significantly from the measured discomfort. For an individual subject, the optimal value was assumed to be when the predicted discomfort changed from being greater than the measured discomfort to being less than the measured discomfort. Over the group of subjects, or for any individual subject, the optimal value was considered to be when the $p$ value in the Wilcoxon matched-pairs signed ranks test comparing predicted and measured values reached the maximum value of 1.0 .

\subsubsection{Masking methods}

With dual-axis vibration, it is assumed that the discomfort caused by a secondary component is reduced because it is 'masked' by the discomfort caused by the most uncomfortable component (Griffin and Whitham, 1977). In the summation procedure, the two discomforts are added but the discomfort of the secondary component is multiplied by a masking coefficient with a value between 0 and 1 . With tri-axial vibration, this may be expressed as:

$$
\psi_{\text {total }}=\psi_{1}+A \cdot \psi_{2,3}
$$


where $\Psi_{\text {total }}$ is the predicted discomfort of the multi-axis vibration, $\Psi_{1}$ is the discomfort of the most uncomfortable component, $\Psi_{2,3}$ is the discomfort caused by the two secondary components, and $A$ is the masking coefficient.

The discomfort caused by the two secondary components is mainly determined by the most uncomfortable of the two (of subjective magnitude $\Psi_{2}$ ), as the effect of the smallest component is reduced by a similar masking effect. It is assumed that the masking coefficient, $A$, is the same because the masking effect is similar:

$$
\psi_{2,3}=\psi_{2}+A \cdot \psi_{3}
$$

As a consequence:

$$
\psi_{\text {total }}=\psi_{1}+A \cdot \psi_{2}+A^{2} \cdot \psi_{3}
$$

Where $\Psi_{\text {total }}$ is the predicted discomfort of the multi-axis vibration and $\Psi_{1}, \Psi_{2}$, and $\Psi_{3}$ are the subjective magnitudes of, respectively, the most uncomfortable component, the second most uncomfortable component, and the least uncomfortable component.

If $A=0$, secondary components are completely masked by the main component and the model is equivalent to the 'worst component' model. If $A=1$, no masking occurs and discomfort is predicted by the linear sum of the discomfort caused by all components.

A value of the masking coefficient, $A$, in the range 0 to 1 can be expected, at which there is no significant difference between the predicted and measured discomfort caused by multi-axis vibration. This value was determined in a similar way to the optimal exponent for the power summation method (Section 2.6.1). 


\section{Results}

\subsection{Worst component methods and linear sum}

At both frequencies and for all subjects, the method of linear sum (i.e., $\alpha=0$ or $A=1$ ) overestimated the vibration discomfort $(p<0.05)$. The method of the worst component (i.e., $\alpha=\infty$ or $A=0$ ) significantly underestimated the discomfort for 11 subjects at $1 \mathrm{~Hz}$, and for 14 subjects at $4 \mathrm{~Hz}(p<0.05)$. These results are consistent with the assumptions in Sections 2.6.1 and 2.6.2.

\subsection{Optimal summation method}

The $p$-values obtained when comparing the measured discomfort with the discomfort predicted using Equation (1) and $\alpha$ in the range 1 to 4 are shown in Figure 2 for $1-\mathrm{Hz}$ vibration and $4-\mathrm{Hz}$ vibration.

\section{FIGURE 2 AROUND HERE}

For small values of the exponent $\alpha(\alpha<2.56$ at $1 \mathrm{~Hz}, \alpha<2.82$ at $4 \mathrm{~Hz})$, the predicted discomfort was significantly greater than the measured discomfort $(p<0.05)$. For high values of $\alpha(\alpha>2.88$ at $1 \mathrm{~Hz}, 3.14$ at $4 \mathrm{~Hz})$, the predicted discomfort was significantly less than the measured discomfort $(p<0.05)$.

The ranges of values for $\alpha$ for which the predictions were not significantly different from the measured discomfort are shown in Table 2 and as shaded areas in Figure 2.

\section{TABLE 2 AROUND HERE}

The results suggest that values of $\alpha$ between 2.6 and 2.9 may be optimum for $1-\mathrm{Hz}$ vibration, and values of $\alpha$ between 2.8 and 3.1 may be optimum for $4-\mathrm{Hz}$ vibration. 
Figure 3 illustrates the relationship between the subjective magnitudes reported by subjects and the subjective magnitudes predicted with the power summation method using the optimal exponents (i.e. 2.7 at $1 \mathrm{~Hz}$ and 3.0 at $4 \mathrm{~Hz}$ ).

\section{FIGURE 3 ABOUT HERE}

\subsection{Masking method}

Values of the masking coefficient in the range 0 to 1 that resulted in no significant difference from the reported discomfort (i.e., $p>0.05$, Wilcoxon) are shown as shaded areas in Figure 4.

\section{FIGURE 4 ABOUT HERE}

The ranges of $A$-values, as used in Equations 2, 3 and 4, for which the predicted discomfort was not significantly different from the reported discomfort are indicated in Table 2. With $A=0.19$, the discomfort of the multi-axis motions, $\Psi_{\text {total }}$, was well predicted with both frequencies of vibration:

$$
\psi_{\text {total }}=\psi_{1}+0.19 \cdot \psi_{2}+0.19^{2} \cdot \psi_{3}
$$

where $\Psi_{1}, \Psi_{2}$, and $\Psi_{3}$ are the subjective magnitudes of the most uncomfortable, the second most uncomfortable, and the least uncomfortable component, respectively.

\subsection{The effect of different relative magnitudes between components}

Some of the test stimuli consisted of three components of similar equivalent subjective magnitude while other stimuli had a main component much more uncomfortable than the other components. A masking coefficient might be suitable for a limited range of relative magnitudes between components. The optimal methods may provide a useful overall average for the selection of stimuli presented in this study but, for example, underestimate homogeneous stimuli and overestimate highly heterogeneous stimuli. 
The motion stimuli were characterized by their heterogeneity, $H$, the ratio of the discomfort caused by the worst component to the mean of the discomfort caused by the three components:

$$
H=\frac{\max \left(\psi_{x}, \psi_{y}, \psi_{z}\right)}{\frac{1}{3}\left(\psi_{x}+\psi_{y}+\psi_{z}\right)}
$$

If the three components produced similar discomfort, $H$ was close to 1 . If two of the components were negligible compared to a dominant component, $H$ was close to 3 . Examples of tri-axial motions with heterogeneities 1, 1.5, 2, 2.5, and 3 are shown in Figure 5. These examples were created to demonstrate the concept of heterogeneity and were not necessarily motion stimuli used in the experiment.

\section{FIGURE 5 AROUND HERE}

The logarithmic error between the measured discomfort and the discomfort predicted by the power summation model with four different values of the exponent $\alpha$, are shown as a function of heterogeneity, $H$, in Figures 6 and 7 . The test stimuli were also sorted into four groups according to their heterogeneity $(1<H<1.5 ; 1.5<H<2 ; 2<H<2.5 ; 2.5<H<3)$ and the median error and interquartile range were calculated for each group.

\section{FIGURE 6 AROUND HERE}

\section{FIGURE 7 AROUND HERE}

The error in the predicted discomfort for stimuli with a low heterogeneity (i.e., motions for which the three components are similar) depends on the exponent, $\alpha$. As expected, for these stimuli the discomfort was overestimated by the linear sum and underestimated by the 'worst component' to a greater extent than motions with one dominant component. When $\alpha=2.85$, the heterogeneity, $H$ had little effect. 
The effect of $H$ on the prediction error is compared between the power summation method (with $\alpha=2.85$ ) and the masking method (with $A=0.19$ ) with data pooled for both frequencies in Figure 8. A systematic trend is apparent with the masking method: motions with $H>2$ were, on average, slightly overestimated while motions with $H<1.5$ were underestimated. Although the median errors are small in both cases, the power summation method may be preferred as the coefficient is less dependent on the choice of test stimuli. Motions with $H>2.5$ tended to be overestimated by both methods, but this may have resulted from a small number of test stimuli.

\section{FIGURE 8 AROUND HERE}

It may be concluded that an exponent of about 2.85 is applicable to multi-axis motions with different relative subjective magnitudes between components and that it is not the result of the choice of multi-axis vibration stimuli used in the experiment.

\subsection{The effect of stimulus magnitude}

If the error in predicting vibration discomfort is defined as:

$$
\text { error }=\log \left(\Psi_{\text {predicted }} / \Psi_{\text {measured }}\right)
$$

the error will be positively correlated with $\boldsymbol{\Psi}_{\text {predicted }}$ and negatively correlated with $\Psi_{\text {measured. }}$ To investigate the effect of vibration magnitude on the prediction error, an unbiased estimate of the subjective magnitudes of the stimuli was chosen:

$$
\psi_{m}=\frac{\psi_{\text {measured }}+\psi_{\text {predicted }}}{2}
$$

The prediction error obtained using the power summation model with $\alpha=2.85$ is shown in Figure 9 as a function of $\Psi_{\mathrm{m}}$. For the subjective magnitudes grouped in six categories $\left(\Psi_{m}=0-50,50-100,100-150,150-200,200-250\right.$, and $\left.250-300\right)$ the median and inter-quartile range of the prediction error are also shown in Figure 8. 


\section{FIGURE 9 AROUND HERE}

At both frequencies, and with all categories of stimuli, the median prediction error is close to zero with no systematic effect of subjective magnitude. This suggests that the optimal prediction method was suitable for the whole range of subjective magnitudes and not a result of the choice of vibration stimuli used.

\section{Discussion}

\subsection{Power summation methods in the literature}

\subsubsection{Standardised method}

In International Standard 2631-1:1974 (now obsolete), it was recommended that the discomfort of multi-axis vibration should be estimated from the discomfort of the most severe single-axis component. This 'worst component method' was changed by an amendment in 1982 and, thereafter, ISO 2631 has recommended that the root-sum-ofsquares of the weighted accelerations in all axes should be used when evaluating vibration with respect to comfort:

$$
a_{v}=\left(k_{x}^{2} \cdot a_{w x}^{2}+k_{y}^{2} \cdot a_{w y}^{2}+k_{z}^{2} \cdot a_{w z}^{2}\right)^{1 / 2}
$$

where $a_{w x}, a_{w y}$, and $a_{w z}$ are the frequency-weighted r.m.s. accelerations in the fore-andaft, lateral, and vertical directions, respectively, and $k_{\mathrm{x}}, k_{\mathrm{y}}$, and $k_{\mathrm{z}}$ are multiplying factors that take into account human sensitivity to vibration in each axis.

\subsubsection{Previous studies}

Methods of predicting the discomfort of seated people exposed to dual-axis vibration (combined lateral and vertical $3.15-\mathrm{Hz}$ sinusoidal vibration) from the discomfort of the 
single-axis components of the motion were investigated by Griffin and Whitham (1977). The discomfort produced by the multi-axis and single-axis stimuli was measured by the 'equivalent magnitude' of a single axis motion (either vertical or lateral) that caused equivalent discomfort. The discomfort of the multi-axis stimuli was therefore predicted from the physical magnitudes of the single-axis components and not their subjective magnitudes (i.e., discomfort). It was concluded that the linear sum of equivalent component magnitudes overestimated the discomfort of dual-axis vibration. The masking model, when optimized, fitted the data slightly better than the root-sum-of-squares, which was an expected result of the optimization of the masking parameters. However the root-sum-of-squares provided satisfying predictions and was considered more suitable for practical use.

A similar method has been used to investigate the discomfort of seated subjects exposed to dual-axis (fore-and-aft and vertical, and lateral and vertical) $3.15-\mathrm{Hz}$ and 6.3-Hz sinusoidal vibration (Mistrot et al., 1990). The predictions with power-summation methods differed from the experimental results with exponents of $1,3,4,5$ and $\infty$, but with an exponent of 2 there was no significant difference. A similar approach was taken by Griefahn and Bröde (1999) who found that an exponent of 1 or 2 was better than an exponent of 3, suggesting the most appropriate exponent was between 1 and 2 .

A different approach was taken by Fairley and Griffin (1988) who predicted the discomfort of seated subjects exposed to dual-axis (fore-and-aft and vertical) vibration from the discomfort (i.e., the subjective magnitudes) caused by the two component motions rather than from their physical magnitudes, and assumed a power summation method could be used to predict the discomfort, similar to Equation (1). The optimal exponent was found to be around 2, with no large difference when the vibration frequency varied from $2.5 \mathrm{~Hz}$ to $10 \mathrm{~Hz}$. The linear sum method overestimated, and the 
'worst component' method underestimated, the discomfort caused by dual-axis vibration. Ratios between subjective magnitudes in the two axes varied from 1:9 to 9:1, similar to the present experiment where the acceleration ratios between components varied from 1:8 to 8:1. However, unlike the present experiment where the discomfort of each singleaxis components was obtained by magnitude estimation, Fairley and Griffin (1988) estimated the discomfort of single-axis components assuming discomfort was linearly related to vibration magnitude.

The discomfort, $\psi$, produced by sinusoidal vibration varies as a function of acceleration magnitude, $\varphi$, according to a power law:

$$
\Psi=k \varphi^{n}
$$

For the whole-body vibration of standing people, the value of $n$ has been reported to be around 0.7 (Thuong and Griffin, 2011a).

When predicting the discomfort of multi-axis vibration from the single-axis components, the exponent of 2 has been found for summing the weighted accelerations (i.e., physical magnitudes) but not for summing the discomfort (i.e., subjective magnitudes; Mistrot et al, 1990).

If the discomfort, $\psi$, caused by a vibration stimulus is expressed in terms of the 'equivalent magnitude' (i.e., the magnitude of a reference vibration that causes equivalent discomfort), and the total discomfort of a multi-axis stimulus ( $a_{\text {total,eq }}$ ) can be predicted from the three equivalent accelerations using a power summation method with a power (i.e., exponent) $\beta$, then:

$$
a_{\text {total }, e q}=\left(a_{x, e q}^{\beta}+a_{y, e q}^{\beta}+a_{z, e q}^{\beta}\right)^{1 / \beta}
$$


Since $\psi_{\text {total }}$ is proportional to $a_{\text {total,eq }}{ }^{n}$ and $a_{x, \text { eq }}$ is proportional to $\psi_{x}$ (and similarly for other axes), it can then be shown that the subjective magnitude for a multi-axis vibration can be predicted from:

$$
\psi_{\text {total }}=\left\{\left(\psi_{x}\right)^{\frac{\beta}{n}}+\left(\psi_{y}\right)^{\frac{\beta}{n}}+\left(\psi_{z}\right)^{\frac{\beta}{n}}\right\}^{\frac{n}{\beta}}
$$

So the rate of growth of discomfort in the reference vibration, $n$, affects the exponent, $\alpha$, in the summation procedure:

$$
\alpha=\beta / n
$$

\section{$\underline{4.1 .3 \text { Comparison of present results with previous studies }}$}

In the study by Griffin and Whitham (1977), the adjustable stimulus was a $3.15-\mathrm{Hz}$ vertical or lateral vibration, whereas in the study by Mistrot et al. (1990), the adjustable stimulus was a $3.15-\mathrm{Hz}$ or $6.3-\mathrm{Hz}$ fore-and-aft or lateral vibration. In those conditions, the median rate of growth of sensation $n$ is expected to vary from 0.50 to 0.95 for the adjustable vibration, due to variations in the value of $n$ with the frequency and direction of vibration (Morioka and Griffin, 2006). Assuming $\alpha=2.85$ (see Equation 1), the optimal value in the present study, the value of $\beta$ fell between $1.4(\beta=2.85 \times 0.50)$ and $2.7(\beta=$ 2.85x0.95) (Equation 13). Despite the differences in the stimuli and the experimental methods, this is consistent with the value of $\beta=2$ recommended in those studies, in which integer values of $\beta$ were privileged.

\subsection{Predicting the discomfort of multi-axis vibration}

The experimental results showed that the discomfort of a multi-axis vibration could be predicted with no bias when using either a power summation model or a masking model:

$$
\psi_{\text {total }}=\left(\psi_{x}^{2.85}+\psi_{y}^{2.85}+\psi_{z}^{2.85}\right)^{1 / 2.85}
$$




$$
\psi_{\text {total }}=\psi_{1}+0.19 \cdot \psi_{2}+0.19^{2} \cdot \psi_{3}
$$

The masking model seemed to require calibration to suit particular relative magnitudes of components, although the effect was small (Section 4.1). The power summation model was equally suitable for all relative magnitudes and the prediction error was not dependent on the subjective magnitude of the vibration stimuli (Section 4.2). These results suggest that the optimized power summation model was not the result of the choice of vibration magnitudes or the composition of the multi-axis stimuli, and that it is applicable in a broader range of situations.

For practical applications, it is convenient to predict vibration discomfort from the physical magnitudes of the components of a vibration, as their subjective magnitudes are not known. As shown in Equation (13), the required exponent, $\beta$, is equal to the exponent $\alpha$ multiplied by the rate of growth of sensation, $n$. The rate of growth depends on the direction and the frequency of vibration (Thuong and Griffin, 2011a). For standing people exposed to vibration in the frequency range 0.5 to $16 \mathrm{~Hz}, n$ varies between about 0.4 and 1.5 , with an average value of 0.72 . This suggests that although $\beta$ should also depend on the frequency and direction of vibration, the vibration discomfort associated with multi-axis multi-frequency vibration might be estimated using a value of $\beta$ calculated from the average value of $n$ (i.e., 0.72) and the optimal value of the exponent $\alpha$ (i.e., 2.85):

$$
\beta=0.72 * 2.85=2.05
$$

Considering the wide variability in $n$, the more practical value of 2.0 for $\beta$ seems appropriate when estimating vibration discomfort from the physical magnitudes of the three components of tri-axial vibration:

$$
\psi_{\text {total }}=\left(\left(k_{x} \cdot a_{w x}\right)^{2}+\left(k_{y} \cdot a_{w y}\right)^{2}+\left(k_{z} \cdot a_{w z}\right)^{2}\right)^{1 / 2}
$$


A frequency weighting is required for each axis (Thuong and Griffin, 2011a) to obtain the three frequency-weighted components $\left(a_{w x}, a_{w y}, a_{w z}\right)$, which are then adjusted according to differences in sensitivity between the three axes (Thuong and Griffin, 2012) using multiplying factors $\left(k_{\mathrm{x}}, k_{\mathrm{y}}, k_{\mathrm{z}}\right)$, before being summed to predict the overall vibration discomfort of the multi-axis multiple frequency vibration.

\section{Conclusion}

Although not optimum for all vibration stimuli, when standing people are exposed to multi-axis random vibration, it seems reasonable to estimate their vibration discomfort from the root-sums-of-squares of the frequency-weighted vibration magnitudes in each of the three directions of translational vibration, as recommended in standards.

No great difference in the optimum method of predicting the discomfort of multi-axis vibration was found between $1-\mathrm{Hz}$ vibration and $4-\mathrm{Hz}$ vibration, although these two frequencies cause the discomfort of standing people by different mechanisms (Thuong and Griffin, 2011a).

A model for combining the discomfort caused by each axis of vibration was found to be applicable to multi-axis stimuli with different total magnitudes and different relative magnitudes between components. However, the root-sum-of-squares of the frequencyweighted vibration magnitudes in each axis will not provide an optimal prediction of discomfort for all stimuli because the rate of growth of discomfort with increasing magnitude of vibration depends on the frequency and the direction of vibration excitation.

\section{Acknowledgements}

This study was conducted with the support of Société Nationale des Chemins de fer Français (SNCF), Paris, France. The assistance of Cedric Gallais is gratefully 
acknowledged.

\section{References}

British Standards Institution, 1987. BS 6841, Measurement and evaluation of human exposure to whole-body mechanical vibration.

Donati, P., Grosjean, A., Mistrot, P., and Roure, L., 1983. The subjective equivalence of sinusoidal and random whole-body vibration in the sitting position (an experimental study using the floating reference vibration method). Ergonomics 26, pp. 251-273.

Fairley, T. and Griffin, M. 1988. Predicting the discomfort caused by simultaneous vertical and fore-and-aft whole-body vibration. Journal of Sound and Vibration 124, pp. 141-156.

Griefahn, B. and Bröde, P. 1999. The significance of lateral whole-body vibrations related to separately and simultaneously applied vertical motions. A validation study of ISO 2631. Applied Ergonomics 30, pp. 505-513.

Griffin, M.J. and Whitham, E.M. 1977. Assessing the discomfort of dual-axis whole-body vibration. Journal of Sound Vibration 54, pp. 107-116.

Griffin, M.J., Whitham, E.M., Parsons, K.C., 1982. Vibration and comfort I. Translational seat vibration. Ergonomics 25, pp. 603-630.

International Organization for Standardization, 1997. ISO 2631-1, Mechanical vibration and shock - evaluation of human exposure to whole-body vibration - part 1: general requirements.

Mistrot, P., Donati, P., Galmiche, J., Florentin, D., 1990. Assessing the discomfort of the whole-body multi-axis vibration: laboratory and field experiments. Ergonomics 33, pp. 1523-1536. 
Morioka, M. and Griffin, M.J., 2006. Magnitude-dependence of equivalent comfort contours for fore-and-aft, lateral and vertical whole-body vibration. Journal of Sound and Vibration 298, pp. 755-772.

Parsons, K.C. and Griffin M.J., 1988. Whole-body vibration perception thresholds. Journal of Sound and Vibration 121, pp. 237-258.

Pheasant, S., 1988. Bodyspace: anthropometry, ergonomics and design, Taylor \& Francis, London, 1988.

Ruffell, C. and Griffin, M.J., 1995. Effects of 1-Hz and 2-Hz transient vertical vibration on discomfort. The Journal of the Acoustical Society of America 98, p. 2157.

Thuong, O. and Griffin, M.J., 2011a. The vibration discomfort of standing persons: 0.516- $\mathrm{Hz}$ fore-and-aft, lateral, and vertical vibration. Journal of Sound and Vibration 330, pp. 816-826. doi:10.1016/j.jsv.2010.08.040

Thuong, O. and Griffin, M.J., 2011b. The vibration discomfort of standing persons: the effect of body supports. Proceedings of the Institution of Mechanical Engineers, Part F: Journal of Rail and Rapid Transit 225, pp. 228-235.

Thuong, O. and Griffin, M.J., 2011c. The vibration discomfort of standing persons: evaluation of random and transient motions. Ergonomics 54, pp. 1228-1239.

Thuong, O. and Griffin, M.J., 2012. The vibration discomfort of standing people: relative importance of fore-and-aft, lateral and vertical vibration. Applied Ergonomics 43 (5), 902908 . 
Table 1 Unweighted magnitudes of $1-\mathrm{Hz}$ and $4-\mathrm{Hz}$ reference and test vibrations (all magnitudes are in $\mathrm{m} . \mathrm{s}^{-2}$ r.m.s.)

\begin{tabular}{|c|c|c|c|c|c|c|}
\hline & \multicolumn{3}{|c|}{$1 \mathrm{~Hz}$} & \multicolumn{3}{|c|}{$4 \mathrm{~Hz}$} \\
\hline & $\begin{array}{l}\text { Fore- } \\
\text { and-aft }\end{array}$ & Lateral & Vertical & $\begin{array}{l}\text { Fore- } \\
\text { and-aft }\end{array}$ & Lateral & Vertical \\
\hline $\begin{array}{l}\text { Reference } \\
\text { motion }\end{array}$ & 0.15 & 0.15 & 0.30 & 0.30 & 0.30 & 0.20 \\
\hline Magnitude 1 & $\overline{0}$ & $\overline{0}$ & 0 & 0 & $\overline{0}$ & 0 \\
\hline Magnitude 2 & 0.09 & 0.09 & 0.19 & 0.19 & 0.19 & 0.13 \\
\hline Magnitude 3 & 0.15 & 0.15 & 0.30 & 0.30 & 0.30 & 0.20 \\
\hline Magnitude 4 & 0.24 & 0.24 & 0.48 & 0.48 & 0.48 & 0.32 \\
\hline Magnitude 5 & 0.38 & 0.38 & 0.75 & 0.75 & 0.75 & 0.50 \\
\hline
\end{tabular}


Table 2 Suitable ranges (for which $p>0.05)$ and optimal values $(p=1.0)$ of $\alpha$-values (for the power summation model) and $A$-values (for the masking model) obtained with all subjective data with $1-\mathrm{Hz}$ and $4-\mathrm{Hz}$ vibration.

\begin{tabular}{|c|c|c|c|c|}
\hline \multirow{2}{*}{$\alpha$} & & \multicolumn{2}{|c|}{ Suitable range } & Optimal value \\
\hline \multirow{2}{*}{$\alpha$} & $1 \mathrm{~Hz}$ & 2.6 & 2.9 & 2.7 \\
\cline { 2 - 5 } & $4 \mathrm{~Hz}$ & 2.8 & 3.1 & 3.0 \\
\hline \multirow{2}{*}{$A$} & $1 \mathrm{~Hz}$ & 0.19 & 0.23 & 0.21 \\
\cline { 2 - 5 } & $4 \mathrm{~Hz}$ & 0.16 & 0.19 & 0.18 \\
\hline
\end{tabular}




\section{Figure legends}

Figure 1 Photograph and model of the safety frame mounted on the 6-degree-offreedom motion simulator.

Figure 2 The $p$-values of the Wilcoxon matched-pairs signed ranks test comparing the measured discomfort of $1-\mathrm{Hz}$ and $4-\mathrm{Hz}$ vibration with the predictions obtained with all subjects using a power summation method (Equation 1), with values of $\alpha$ between 1.0 and 4.0. The shaded area is the range of $\alpha$ for which the predicted discomfort was not significantly different from the measured discomfort $(p>0.05)$.

Figure 3 Scattergrams showing the relationship between the measured discomfort of 1$\mathrm{Hz}$ and $4-\mathrm{Hz}$ vibration with predictions based on a power summation method (Equation 1) with the optimal values of $\alpha$ (2.7 and 3.0 at $1 \mathrm{~Hz}$ and $4 \mathrm{~Hz}$, respectively). Triangles represent tri-axial stimuli, circles represent dual-axis stimuli.

Figure 4 The $p$-value of the Wilcoxon matched-pairs signed ranks test comparing the measured discomfort of $1-\mathrm{Hz}$ and $4-\mathrm{Hz}$ vibration with the predictions obtained with all subjects using the masking model (Equation 4), with values of $A$ between 0 and 0.5. The shaded area is the range of $A$ for which the predicted discomfort was not significantly different from the measured discomfort $(p>0.05)$.

Figure 5 Examples of tri-axial motions with heterogeneity, $H$, of $1,1.5,2,2.5$, and 3 . (Equation 6).

Figure 6 The effect of the heterogeneity $H$ (Equation 6 ) on the errors in predicting vibration discomfort with the power summation model (Equation 1) with several $\alpha$ values (1- $\mathrm{Hz}$ vibration). Individual data from all subjects. The line and the bars show the medians and the interquartile ranges of the prediction errors for five ranges of heterogeneity. 
Figure 7 The effect of the heterogeneity $H$ (Equation 6) on the errors in predicting vibration discomfort with the power summation model (Equation 1) with several $\alpha$ values (4-Hz vibration). Individual data from all subjects. The line and the bars show the medians and the interquartile ranges of the prediction errors for five ranges of heterogeneity.

Figure 8 Comparison of the effect of heterogeneity on errors in predicting vibration discomfort with the power summation model and the masking model. Individual data from all subjects.

Figure 9 Prediction error (as indicated by Equation 7 when using the power summation model and $\alpha=2.85$ ) as function of the stimulus magnitude $\Psi_{m}$. The line shows the median prediction error for six ranges of unbiased subjective magnitudes $(0-50 ; 50-100$; 100-150; 150-200; 200-250; 250-300). Individual data from all subjects. 


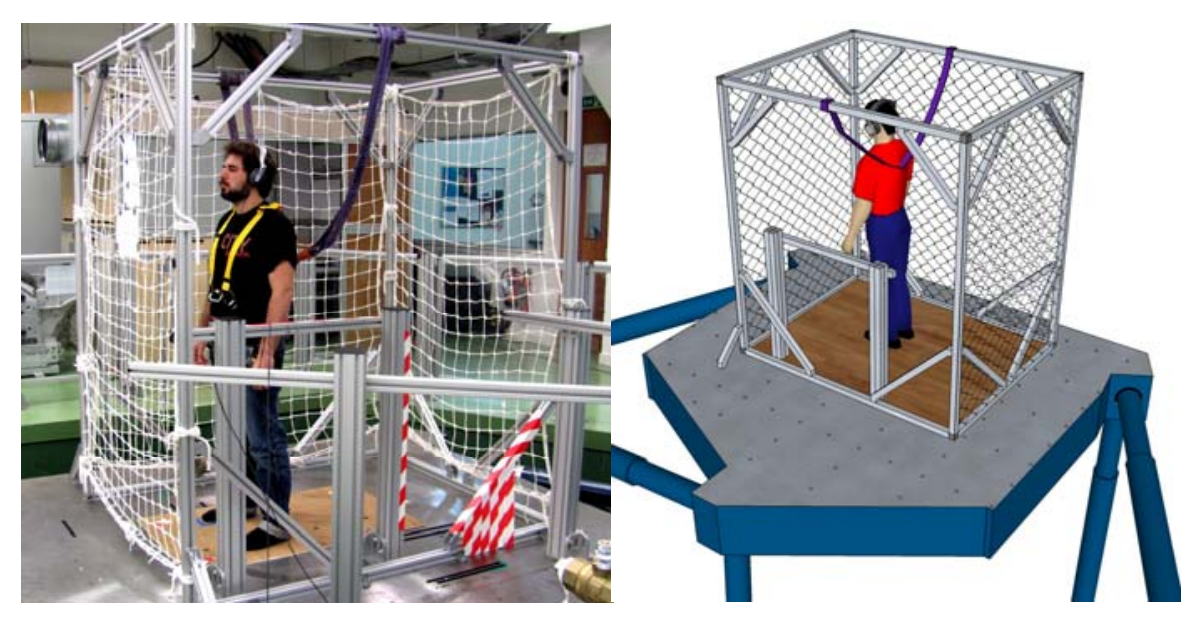

Figure 1 

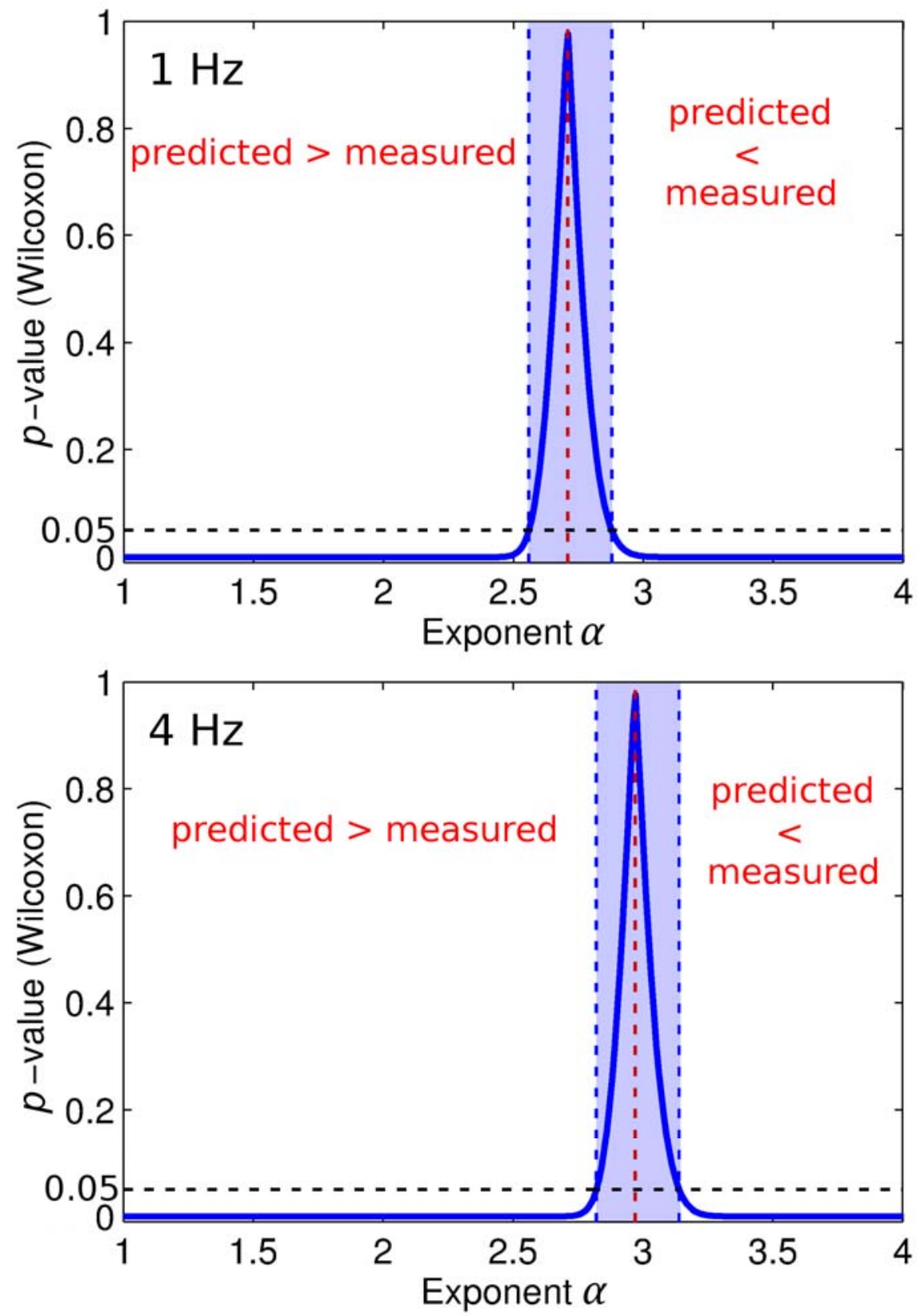

Figure 2 

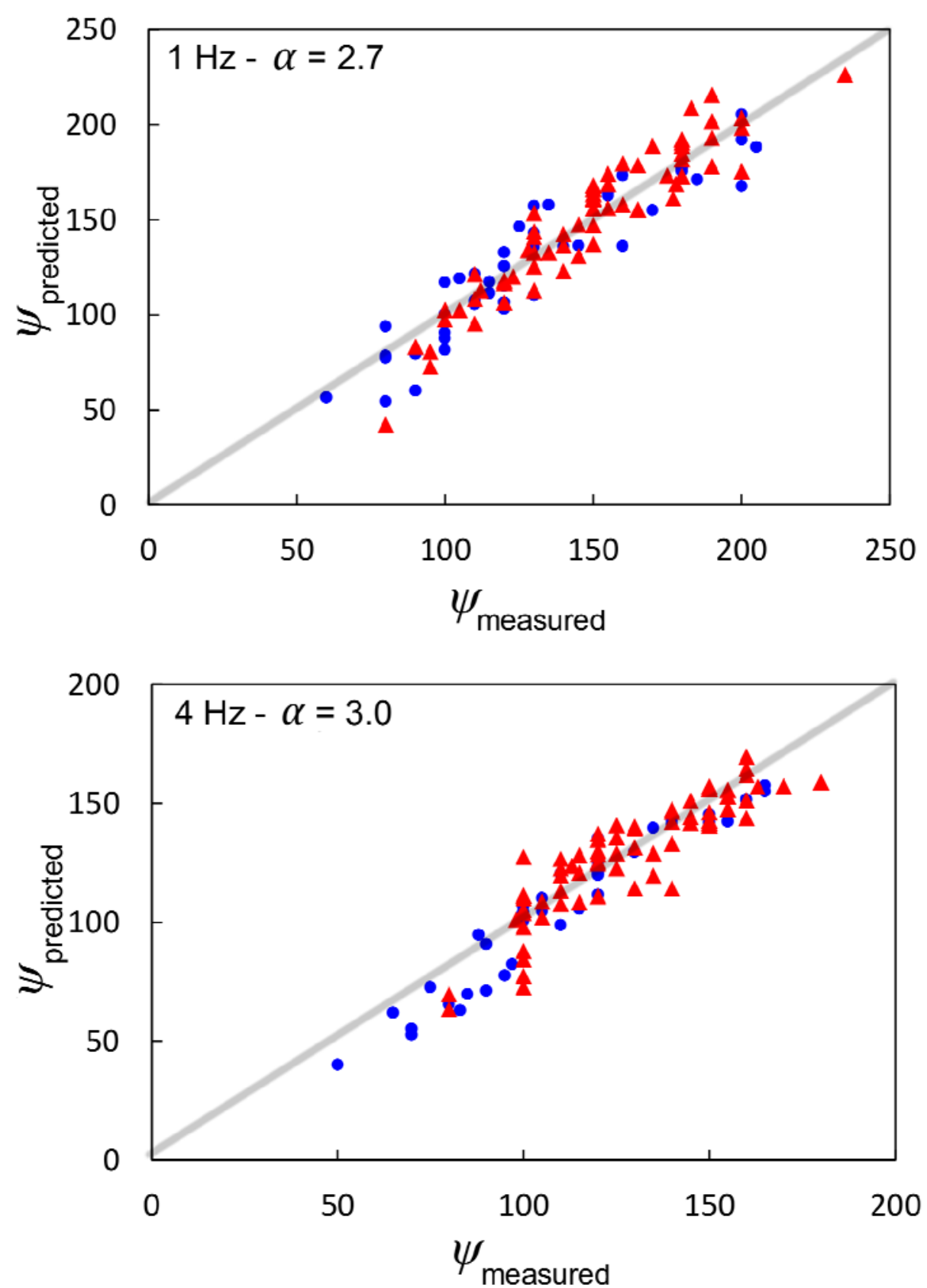

Figure 3 

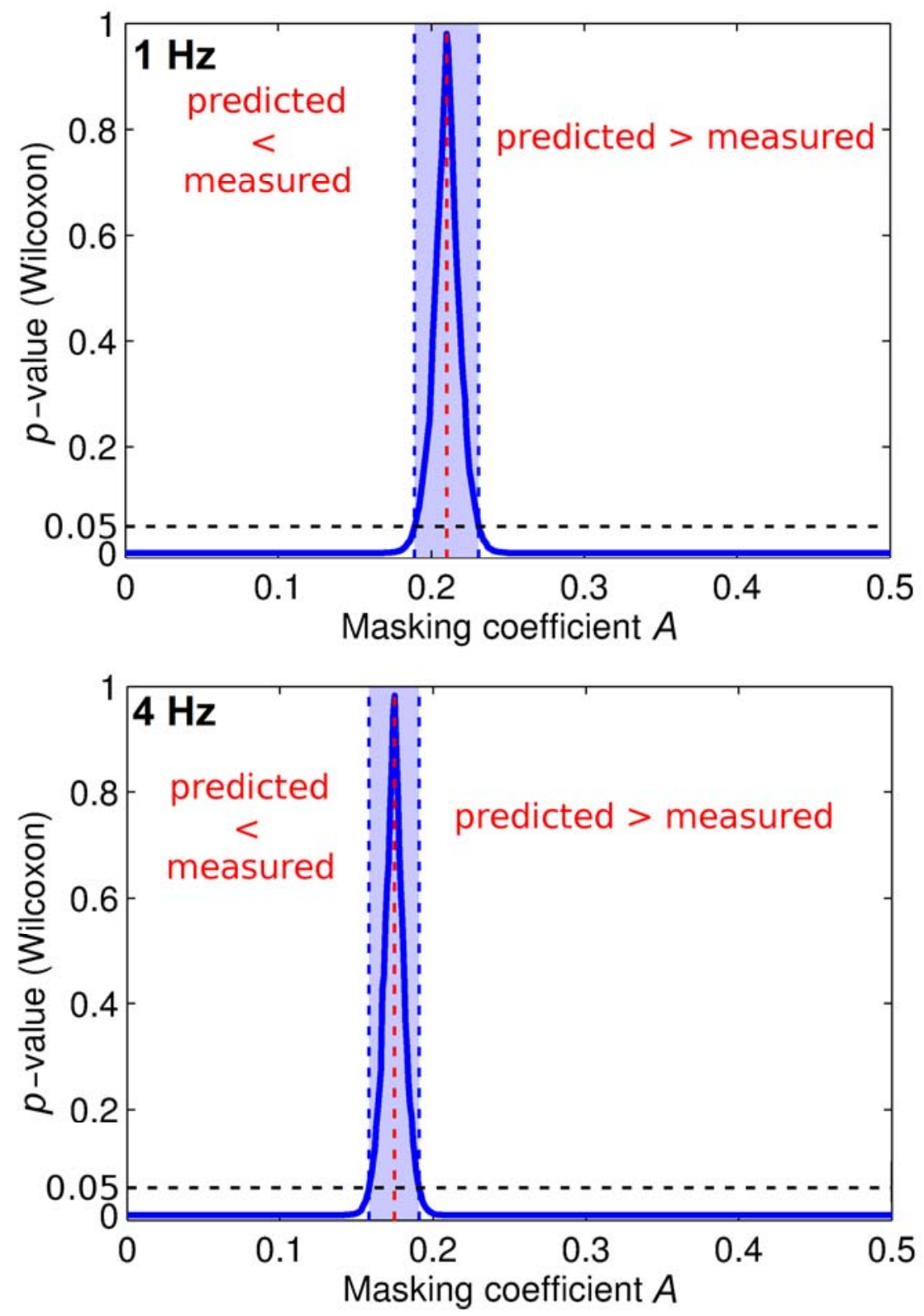

Figure 4 


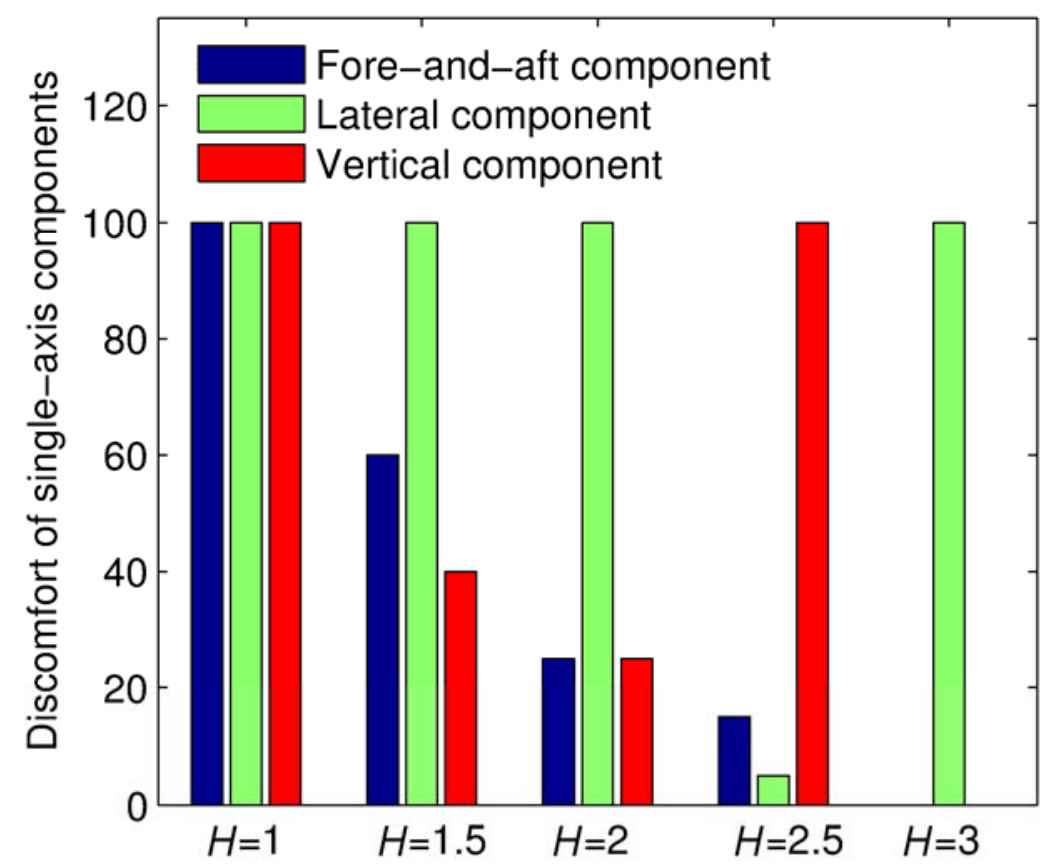

Figure 5 


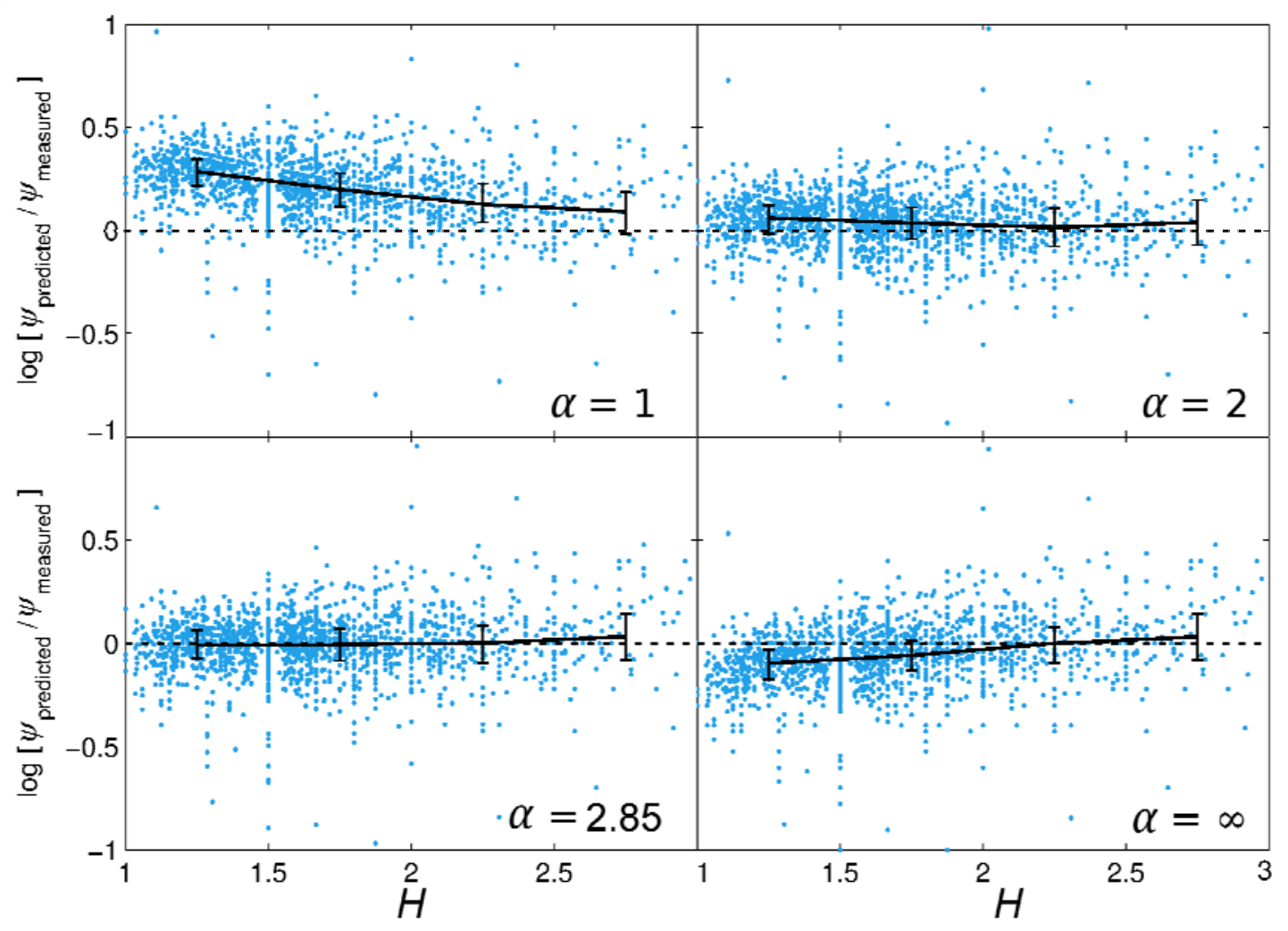

Figure 6 


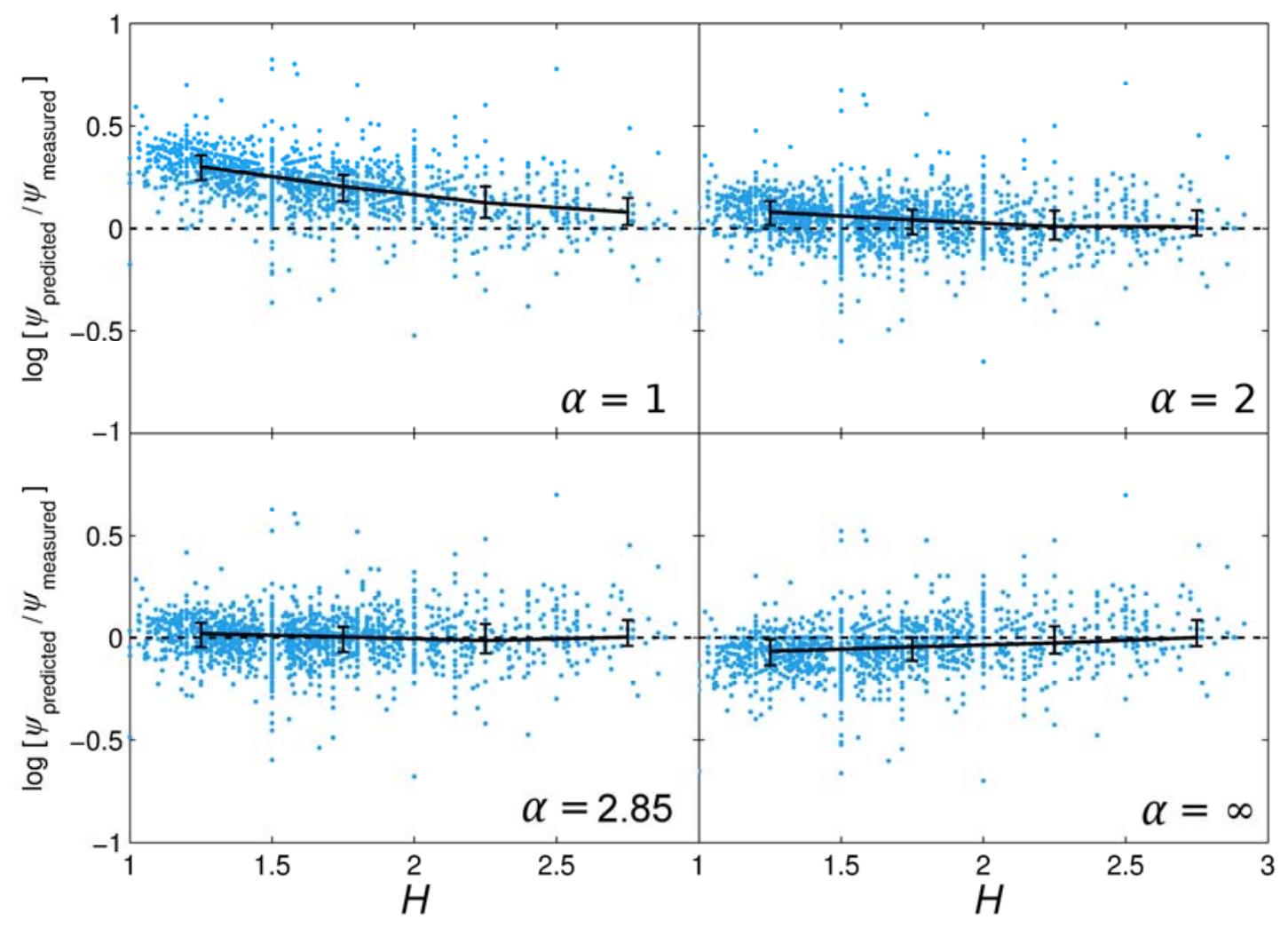

Figure 7 


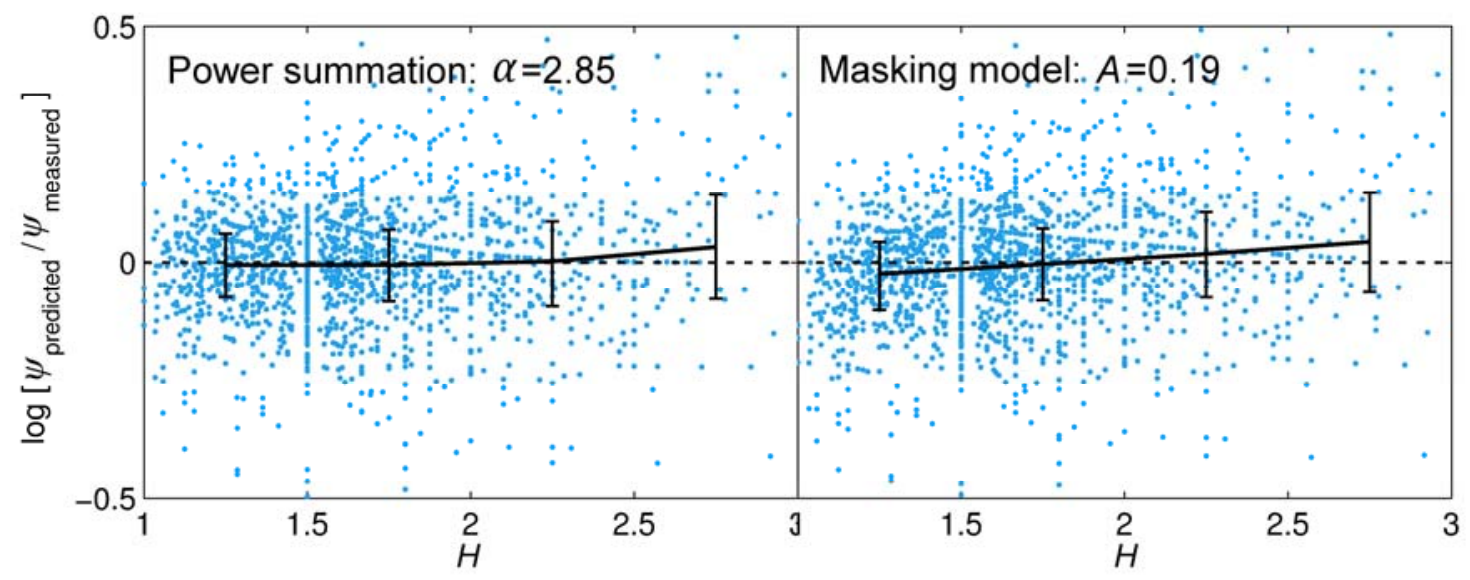

Figure 8 


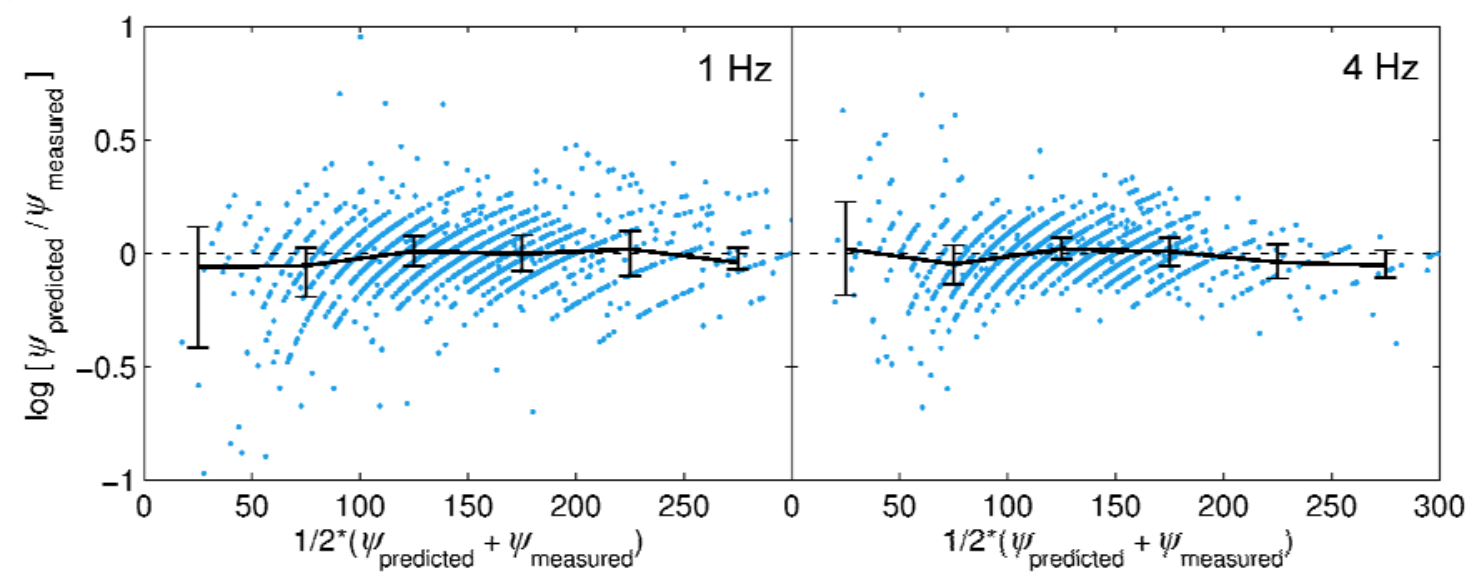

Figure 9 